\title{
Minimizing Trigonometric Matrix Polynomials over Semi-Algebraic Sets
}

\author{
Graziano Chesi \\ Department of Electrical and Electronic Engineering \\ The University of Hong Kong \\ Contact: http://www.eee.hku.hk/ ${ }^{\sim}$ chesi
}

\begin{abstract}
This paper addresses the problem of minimizing the minimum eigenvalue of a trigonometric matrix polynomial. The contribution is to show that, by exploiting Putinar's Positivstellensatz and introducing suitable transformations, it is possible to derive a nonconservative approach based on semidefinite programming (SDP) whose computational burden can be significantly smaller than that of an existing method recently published. Other advantages of the proposed approach include the possibility of taking into account the presence of constraints in the form of semi-algebraic sets and establishing tightness of a found lower bound.
\end{abstract}

\section{Introduction}

Frequency-domain methods are playing a key role in studying control systems since many years. These methods exploit the frequency response of the system, which is the evaluation of the transfer function onto the imaginary axis (continuous-time systems) or onto the complex unit circle (discrete-time systems), see for instance $[1,2]$.

A problem arising in frequency-domain methods consists of minimizing the minimum eigenvalue of a trigonometric matrix polynomial. Indeed, this problem can be met in system modeling, for instance when looking for an approximation of a given systems, and in system design, for instance when imposing bounds of the frequency response of the system.

A possible way of addressing this problem in the case of a scalar variable is through the Kalman-Yakubovich-Popov lemma, see for instance [3]. For the case of multiple variable and scalar trigonometric polynomials, a method 
based on sums of squares (SOS) of trigonometric polynomials has been proposed in [4]. This method has been extended to the case of trigonometric matrix polynomials in [5] where its application to strong stability analysis is described. See also [6] which proposes a simplified method for trigonometric polynomials in two variables and describes its application to FIR filter design.

This paper addresses the problem of minimizing the minimum eigenvalue of a trigonometric matrix polynomial. The contribution is to show that, by exploiting Putinar's Positivstellensatz and introducing suitable transformations, it is possible to derive a nonconservative approach based on semidefinite programming (SDP) whose computational burden can be significantly smaller than that of an existing method recently published. Other advantages of the proposed approach include the possibility of taking into account the presence of constraints in the form of semi-algebraic sets and establishing tightness of a found lower bound. The proposed approach is illustrated by numerical examples which also include an application in the estimation of reduced order models.

The paper is organized as follows. Section 2 introduces the problem formulation and some preliminaries. Section 3 describes the proposed results. Section 4 presents some illustrative examples. Lastly, Section 5 concludes the paper with some final remarks.

\section{Preliminaries}

\subsection{Problem Formulation}

Notation: $\mathbb{N}, \mathbb{Z}, \mathbb{R}, \mathbb{C}$ : sets of natural (including zero), integer, real, and complex numbers; $j$ : imaginary unit; $\Re(A), \Im(A)$ : real and imaginary parts of $A ; \bar{A}$ : complex conjugate of $A$; $I$ : identity matrix (of size specified by the context); $A^{T}, A^{H}$ : transpose and complex conjugate transpose of $A$; Hermitian matrix $A$ : a matrix satisfying $A=A^{H} ; \star$ : corresponding block in Hermitian matrices; $A>0, A \geq 0$ : positive definite and positive semidefinite matrix $A ; \lambda_{\min }(A)$ : minimum real eigenvalue of $A$; matrix polynomial: a matrix whose entries are polynomials; $\operatorname{det}(A)$ : $\operatorname{determinant}$ of $A ; \otimes$ : Kronecker product; $\lfloor a\rfloor$ : largest integer not greater than $a ;\lceil a\rceil$ : smallest integer not smaller than $a ;|a|$ : magnitude of $a ; \bmod (a, b) ; \operatorname{modulo}$ between $a$ and $b$.

Let us denote the unit circle in $\mathbb{C}$ as

$$
\mathcal{T}=\{z \in \mathbb{C}:|z|=1\} .
$$


We say that $F: \mathcal{T}^{n} \rightarrow \mathbb{C}^{m \times m}$ is a trigonometric matrix polynomial if

$$
F(z)=\sum_{k \in \mathcal{S}} F_{k} z^{k}
$$

where $\mathcal{S}$ is a given finite subset of $\mathbb{Z}^{n}, F_{k} \in \mathbb{C}^{m \times m}$ for $k \in \mathcal{S}$ are given matrices, and the notation $z^{k}$ stands for

$$
z^{k}=\prod_{l=1}^{n} z_{l}^{k_{l}} .
$$

Moreover, we say that the trigonometric matrix polynomial $F(z)$ is Hermitian over $\mathcal{T}^{n}$ if

$$
F(z)=F(z)^{H} \quad \forall z \in \mathcal{T}^{n} .
$$

Problem. Let $F: \mathcal{T}^{n} \rightarrow \mathbb{C}^{m \times m}$ and $g_{i}: \mathcal{T}^{n} \rightarrow \mathbb{C}, i=1, \ldots, n_{G}$, be Hermitian trigonometric matrix polynomials over $\mathcal{T}^{n}$. The problem is to solve

$$
\mu^{*}=\min _{z \in \mathcal{G}} \lambda_{\min }(F(z))
$$

where $\mathcal{G} \subseteq \mathcal{T}^{n}$ is the semi-algebraic set

$$
\mathcal{G}=\left\{z \in \mathcal{T}^{n}: g_{i}(z) \geq 0 \quad \forall i=1, \ldots, n_{G}\right\} .
$$

\subsection{SOS Matrix Polynomials}

Here we briefly define SOS matrix polynomials and explain how they can be investigated via LMIs. See also [7-14] and references therein for details. For reasons that will become clear in the next section, we consider matrix polynomials in $2 n$ variables of size $m \times m$.

Let us start by considering the real case. Let $A: \mathbb{R}^{2 n} \rightarrow \mathbb{R}^{m \times m}$ be a matrix polynomial. We say that $A(v), v \in \mathbb{R}^{2 n}$, is $\mathrm{SOS}$ if there exist matrix polynomials $A_{i}: \mathbb{R}^{2 n} \rightarrow \mathbb{R}^{m \times m}, i=1, \ldots, k$, such that

$$
A(v)=\sum_{i=1}^{k} A_{i}(v)^{T} A_{i}(v) .
$$

A necessary and sufficient condition for establishing whether $A(v)$ is SOS can be obtained via an LMI feasibility test. Indeed, $A(v)$ can be expressed as

$$
A(v)=(I \otimes b(v))^{T}(C+L(\alpha))(I \otimes b(v))
$$


where $b(v)$ is a vector of monomials in $v, C$ is a symmetric matrix, and $L(\alpha)$ is a linear parametrization of the linear set

$$
\mathcal{L}=\left\{\tilde{L}=\tilde{L}^{T}:(I \otimes b(v))^{T} \tilde{L}(I \otimes b(v))=0\right\}
$$

with $\alpha$ free real vector. The representation (8) is known as square matrix representation (SMR) and extends the Gram matrix method for (scalar) polynomials to the matrix case. One has that $A(v)$ is SOS if and only if there exists $\alpha$ satisfying the LMI

$$
C+L(\alpha) \geq 0
$$

Next, let us consider the complex case. Let $A: \mathbb{R}^{2 n} \rightarrow \mathbb{C}^{m \times m}$ be a matrix polynomial. We say that $A(v)$ is SOS if there exist matrix polynomials $A_{i}: \mathbb{R}^{2 n} \rightarrow \mathbb{C}^{m \times m}, i=1, \ldots, k$, such that

$$
A(v)=\sum_{i=1}^{k} A_{i}(v)^{H} A_{i}(v) .
$$

Similarly to the real case, this condition holds if and only if there exists $\alpha$ satisfying the LMI

$$
\left(\begin{array}{cc}
\Re(C+L(\alpha)) & \Im(C+L(\alpha)) \\
\star & \Re(C+L(\alpha))
\end{array}\right) \geq 0
$$

where $C$ and $L(\alpha)$ are Hermitian and satisfy (8), in particular $L(\alpha)$ (with $\alpha$ free real vector) is a linear parametrization of the linear set in (9) where $\tilde{L}$ is Hermitian instead of symmetric.

\section{Main Results}

\subsection{Proposed Approach}

Let us express $F(z)$ as in (2), and define its degree as

$$
\operatorname{deg}(F)=\max _{\substack{k \in \mathcal{S} \\ F_{k} \neq 0}} \sum_{l=1}^{n}\left|k_{l}\right| .
$$

Assumption 1. For all $i=1, \ldots, n_{G}, \operatorname{deg}\left(g_{i}\right)$ is even. 
Let us observe that Assumption 1 can be introduced without loss of generality. Indeed, if $\operatorname{deg}\left(g_{i}\right)$ is odd for some $i$, one can redefine such a $g_{i}(z)$ as

$$
g_{i}(z) \rightarrow g_{i}(z) c_{i}(z)
$$

where $c_{i}(z): \mathcal{T}^{n} \rightarrow \mathbb{C}$ is any trigonometric polynomial such that $\operatorname{deg}\left(c_{i}\right)=1$ and

$$
\forall z \in \mathcal{T}^{n}\left\{\begin{array}{l}
c_{i}(z)=c_{i}(z)^{H} \\
c_{i}(z)>0
\end{array}\right.
$$

which ensures that the newly defined trigonometric polynomial has even degree and $\mathcal{G}$ is not modified.

Let us write $z \in \mathcal{T}^{n}$ as

$$
z=x+j y
$$

where $x, y \in \mathbb{R}^{n}$, and define $v \in \mathbb{R}^{2 n}$ as

$$
v=\left(x^{T}, y^{T}\right)^{T} .
$$

Let us express $F(z)$ as in (2), and introduce the matrix polynomial

$$
\Delta(F, v)=\sum_{k \in \mathcal{S}} F_{k} \prod_{\sigma=1}^{n} \delta(v, k, \sigma)
$$

where

$$
\delta(v, k, \sigma)= \begin{cases}\left(v_{\sigma}+j v_{\sigma+n}\right)^{k_{\sigma}} & \text { if } k_{\sigma} \geq 0 \\ \left(v_{\sigma}-j v_{\sigma+n}\right)^{k_{\sigma}} & \text { otherwise. }\end{cases}
$$

Let us define the set

$$
\mathcal{D}= \begin{cases}\mathbb{R} & \text { if } \Delta(F, v), \Delta\left(g_{1}, v\right), \ldots, \Delta\left(g_{n_{G}}, v\right) \text { are real } \\ \mathbb{C} & \text { otherwise. }\end{cases}
$$

Let $A: \mathbb{R}^{2 n} \rightarrow \mathcal{D}^{m \times m}$ be a matrix polynomial expressed as

$$
A(v)=\sum_{k \in \mathbb{N}^{2 n}} A_{k} v^{k}
$$

for some $A_{k} \in \mathcal{D}^{m \times m}$, and introduce

$$
\Theta(A, v)=\sum_{k \in \mathbb{N}^{2 n}} A_{k} \prod_{\sigma=1}^{2 n} \theta(v, k, \sigma)
$$


where

$$
\begin{gathered}
\theta(v, k, \sigma)= \\
\begin{cases}v_{\sigma}^{k_{\sigma}} & \text { if } \sigma \leq n \text { or } k_{\sigma} \leq 2 \\
\left(1-v_{\sigma-n}^{2}\right)^{\left\lfloor k_{\sigma} / 2\right\rfloor} v_{\sigma}^{\bmod \left(k_{\sigma}, 2\right)} & \text { otherwise. }\end{cases}
\end{gathered}
$$

Let us observe that $\Theta(A, v)$ is a matrix polynomial obtained by limiting to 2 the degrees in the variables $v_{n+1}, \ldots, v_{2 n}$ while preserving the equivalence with $A(v)$ over $\mathcal{T}^{n}$. Lastly, let us define the polynomials

$$
s_{l}(v)=1-v_{l}^{2}-v_{l+n}^{2} \quad \forall l=1, \ldots, n .
$$

Theorem 1 One has

$$
F(z)>0 \quad \forall z \in \mathcal{G}
$$

if and only if there exist $\mu>0$ and Hermitian matrix polynomials $P_{i}, Q_{l}$ : $\mathbb{R}^{2 n} \rightarrow \mathcal{D}^{m \times m}, i=1, \ldots, n_{G}$ and $l=1, \ldots, n$, such that

$$
A(v), P_{1}(v), \ldots, P_{n_{G}}(v) \text { are } S O S
$$

where

$$
A(v)=\Theta\left(A_{0}, v\right)
$$

and

$$
\begin{aligned}
A_{0}(v)= & \Delta(F, v)-\mu I-\sum_{i=1}^{n_{G}} \Delta\left(g_{i}, v\right) P_{i}(v) \\
& -\sum_{l=1}^{n} s_{l}(v) Q_{l}(v) .
\end{aligned}
$$

Moreover, without loss of generality (i.e., without introducing conservatism), $P_{i}(v)$ can be chosen to contain only monomials $v^{k}$ that satisfy

$$
k_{\sigma} \leq 2 \quad \forall \sigma=n+1, \ldots, 2 n
$$

and $Q_{l}(v)$ can be chosen to contain only monomials $v^{k}$ that satisfy

$$
\left\{\begin{array}{l}
k_{\sigma} \leq 2 \quad \forall \sigma=n+1, \ldots, 2 n \\
k_{l+n}=0 .
\end{array}\right.
$$

Consequently, the Gram matrices of the matrix polynomials in (26) can be built with respect to vector of monomials $v^{k}$ that satisfy

$$
k_{\sigma} \leq 1 \quad \forall \sigma=n+1, \ldots, 2 n .
$$


Proof. " $\Leftarrow$ " Suppose that $(26)-(28)$ hold. It follows that $A(v), P_{1}(v), \ldots, P_{n_{G}}(v)$ are positive semidefinite for all $v \in \mathbb{R}^{2 n}$. Let us observe that $F(z)=\Delta(F, v)$ and $g_{i}(z)=\Delta\left(g_{i}, v\right)$. Moreover,

$$
z \in \mathcal{G} \Longleftrightarrow v \in \mathcal{V}
$$

where

$$
\begin{aligned}
\mathcal{V}= & \left\{v \in \mathbb{R}^{2 n}: \Delta\left(g_{i}, v\right) \geq 0\right. \text { and } \\
& \left.s_{l}(v)=0 \forall i=1, \ldots, n_{G} \forall l=1, \ldots, n\right\} .
\end{aligned}
$$

Since $A(v)=A_{0}(v)$ for all $v \in \mathcal{V}$, it follows that $A_{0}(v) \geq 0$ for all $v \in \mathcal{V}$. This implies that $F(z)>0$ for all $z \in \mathcal{G}$.

" $\Rightarrow$ " Suppose that (25) holds. Let us observe that $\mathcal{G}$ is compact because bounded (being a subset of $\mathcal{T}^{n}$ ) and closed (since $\mathcal{T}^{n}$ is closed and the polynomial inequalities in (6) are not strict). Hence, it follows that there exists $\mu_{1}>0$ such that

$$
F(z) \geq \mu_{1} \quad \forall z \in \mathcal{G} .
$$

Following the previous part of the proof, it follows that

$$
\Delta(F, v) \geq \mu_{1} \quad \forall v \in \mathcal{V} .
$$

Analogously to $\mathcal{G}$, one has that $\mathcal{V}$ is compact. Moreover, the polynomials $\Delta\left(g_{i}, v\right)$ and $s_{l}(v)$ have even degree and their highest degree homogeneous parts do not have common zeroes in $\mathbb{R}^{2 n}$ except 0 . From Putinar's Positivstellensatz, see Theorem 1.4 in [15], it follows that there exist $\mu>0$ and matrix polynomials $P_{i}(v)$ and $Q_{l}(v)$ such that $A_{0}(v)$ and $P_{1}(v), \ldots, P_{n_{G}}(v)$ are SOS.

Next, let us show that, through substitutions $v_{l+n}^{2}=1-v_{l}^{2}$, it is possible to transform $A_{0}(v)$ into a SOS matrix polynomial whose monomials $v^{k}$ satisfy (29). Since $A_{0}(v)$ is SOS, one can write

$$
A_{0}(v)=\sum_{i=1}^{k} A_{0 i}(v)^{H} A_{0 i}(v)
$$

for some matrix polynomials $A_{0 i}: \mathbb{R}^{2 n} \rightarrow \mathcal{D}^{m \times m}$. Let us define

$$
A_{1}(v)=\sum_{i=1}^{k} A_{1 i}(v)^{H} A_{1 i}(v)
$$

where $A_{1 i}(v)$ is obtained from $A_{0 i}(v)$ with the transformation (22)-(23) where the condition $k_{\sigma} \leq 2$ is replaced by $k_{\sigma} \leq 1$. It follows that $A_{1}(v)$ is SOS and

$$
A_{1}(v)=A_{0}(v) \quad \forall v \in \mathcal{V} .
$$


Moreover, there exist matrix polynomials $\tilde{Q}_{l}(v)$ such that

$$
A_{1}(v)=A_{0}(v)+\sum_{l=1}^{n} s_{l}(v) \tilde{Q}_{l}(v) .
$$

Since the monomials $v^{k}$ of $A_{1}(v)$ satisfy (29), one has

$$
\Theta\left(A_{1}, v\right)=A_{1}(v)
$$

which implies that there exist $\mu>0$ and matrix polynomials $P_{i}(v)$ and $Q_{l}(v) \rightarrow Q_{l}(v)+\tilde{Q}_{l}(v)$ such that $A(v)$ is SOS. In particular, its Gram matrix can be built with respect to vector of monomials of the form $v^{k}$ where each $k$ satisfies (31). The same procedure also shows that $\Theta\left(P_{i}, v\right)$, $i=1, \ldots, n_{G}$, can be assumed SOS, and that their Gram matrices can be reduced according to (31). Lastly, let us observe that $P_{i}(v)$ and $Q_{l}(v)$ can be chosen to satisfy (29)-(30) because the monomials $v^{k}$ that do not satisfy these conditions do not add degrees of freedom to $A(v)$.

Theorem 1 provides an LMI condition for establishing whether (25) holds. This condition is sufficient for any degree of $P_{i}(v)$ and $Q_{l}(v)$, and also necessary for a sufficiently large degree.

This theorem also states that $P_{i}(v)$ and $Q_{l}(v)$ do not need to contain all monomials according to (29)-(30). In particular, the degree on the imaginary part of $z$ (i.e., $v_{n+1}, \ldots, v_{2 n}$ ) is not greater than 2 according to (29) and the first line in (30). Also, the degree on the imaginary part of $z_{l}$ (i.e., $\left.v_{l+n}\right)$ is 0 for $Q_{l}(v)$ according to the second line in (30).

Moreover, the theorem states that the Gram matrices of the Hermitian matrix polynomials in (26) can be built with respect to reduced vectors of monomials according to (31), in particular the degree on the imaginary part of $z$ is not greater than 1 .

In order to clarify with a simple example why $P_{i}(v)$ and $Q_{l}(v)$ do not need to contain all monomials according to (29)-(30), let us consider, for $n=1$ and $m=1, Q_{1}(v)$ of degree 2 parametrized by

$$
Q_{1}(v)=\sum_{\substack{k \in \mathbb{N}^{2} \\ k_{1}+k_{2} \leq 2}} a_{k} v^{k}
$$

where $a_{k} \in \mathbb{R}$. It follows that

$$
\Theta\left(s_{1} Q_{1}, v\right)=s_{1}(v)\left(a_{00}-a_{02}+a_{10} v_{1}+\left(a_{20}+a_{02}\right) v_{1}^{2}\right)
$$


which means that $a_{01}$ and $a_{11}$ do not affect $\Theta\left(s_{1} Q_{1}, v\right)$, while $a_{02}$ can be considered null by redefining $a_{00}$ and $a_{20}$. Hence, the monomials $v_{2}, v_{1} v_{2}$ and $v_{2}^{2}$ do not need to be introduced in $Q_{1}(v)$.

For chosen degree of the matrix polynomials $P_{i}(v)$ and $Q_{l}(v)$, let us define the SDP

$$
\hat{\mu}=\sup _{\mu, P_{i}(v), Q_{l}(v)} \mu
$$

s.t. $(26)-(31)$ hold.

From Theorem 1 it turns out that

$$
\hat{\mu} \leq \mu^{*}
$$

Once that the lower bound $\hat{\mu}$ has been computed, a question arise: is this lower bound tight? In order to answer this question, let us define the function $\xi: \mathbb{R}^{2 n} \rightarrow \mathbb{C}^{n}$ as

$$
\xi(v)=\left(v_{1}+j v_{n+1}, \ldots, v_{n}+j v_{2 n}\right)^{T} .
$$

Theorem 2 One has

$$
\hat{\mu}=\mu^{*}
$$

if and only if there exists $v \in \mathbb{R}^{2 n}$ such that

$$
\left\{\begin{aligned}
\lambda_{\min }(\hat{A}(v)) & =0 \\
\lambda_{\min }(F(\xi(v)) & =\hat{\mu} \\
\xi(v) & \in \mathcal{G}
\end{aligned}\right.
$$

where $\hat{A}(v)$ is the matrix polynomial $A(v)$ evaluated for the optimal values of the variables in (32). Moreover, $\xi(v)$ with such $a v$ is a minimizer of the original optimization problem (5).

Proof. " $\Leftarrow$ " Suppose that $(36)$ holds. Let us define

$$
z=\xi(v)
$$

Since $z \in \mathcal{G}$ and $\lambda_{\min }\left(F(z)=\hat{\mu}\right.$, it follows that $\hat{\mu} \geq \mu^{*}$. From (33) we conclude that (35) holds.

" $\Rightarrow$ " Suppose that (35) holds. Since $\mathcal{G}$ is compact (see proof of Theorem 1 ), it follows that there exists a minimizer $z$ of (5). Define

$$
v=\left(\Re(z)^{T}, \Im(z)^{T}\right)^{T} .
$$


It follows that the second and third conditions in (36) hold since

$$
\xi(v)=z .
$$

Moreover, let $\hat{A}_{0}(v), \hat{P}_{i}(v), \hat{Q}_{l}(v), \hat{\mu}$ be $A_{0}(v), P_{i}(v), Q_{l}(v), \mu$ evaluated for the optimal values of the variables in (32). One has

$$
\begin{aligned}
\hat{A}_{0}(v)= & \Delta(F, v)-\hat{\mu} I=\hat{\mu} I-\sum_{i=1}^{n_{G}} \Delta\left(g_{i}, v\right) \hat{P}_{i}(v) \\
& -\sum_{l=1}^{n} s_{l}(v) \hat{Q}_{l}(v) \\
\leq & \Delta(F, v)-\hat{\mu} I
\end{aligned}
$$

since $\hat{\mu}-0, \Delta\left(g_{i}, v\right) \geq 0, \hat{P}_{i}(v) \geq 0$, and $s_{l}(v)=0$. Since (35) holds and $F(z)=\Delta(F, v)$, one has that

$$
\operatorname{det}(\Delta(F, v)-\hat{\mu} I)=0 .
$$

Let us observe that

$$
\hat{A}_{0}(v)=\hat{A}(v)
$$

because $s_{l}(v)=0$ for all $l=1, \ldots, n$. Moreover,

$$
\hat{A}(v) \geq 0
$$

which implies that the first condition in (36) holds.

Theorem 2 provides a sufficient and necessary condition for establishing whether the lower bound $\hat{\mu}$ is tight based on the construction of a minimizer of the original optimization problem (5). Specifically:

1) one looks for vectors $v$ such that the first condition in (36) holds. Since $\hat{A}(v)$ is positive semidefinite, this is equivalent to the existence of $w \in \mathcal{D}^{m}, w \neq 0$, such that

$$
b(v) \otimes w \in \operatorname{ker}(\hat{C})
$$

where $\hat{C}$ is the Gram matrix of $\hat{A}(v)$ obtained from $(32)$ and $b(v)$ is the corresponding vector of monomials. The computation of such $v$ and $w$ can be addressed via linear algebra as explained in [16] (specifically, via Cholesky factorizations, column echelon forms, and Schur decompositions) or in [17] (specifically, via pivoting operations and computing the roots of univariate polynomials); 
2 ) then, one verifies if, for any of such vectors $v$, the second and third conditions in (36) hold;

3 ) if yes, the lower bound $\hat{\mu}$ is tight and $\xi(v)$ is a minimizer of the original optimization problem (5).

Let us mention that conditions for establishing tightness in optimization over polynomials have been proposed such as the flat truncation (see for instance [18]), which consists of checking whether the rank of the moment matrix stabilizes, and it is always sufficient and almost necessary. With respect to the flat truncation, the condition of Theorem 2 requires some additional computations to determine the minimizer, however this results in a condition that is always necessary and sufficient, moreover it also provides a minimizer of the original optimization problem (5).

\subsection{Numerical Complexity}

Here we compare the numerical complexity of the proposed approach with that of the existing method in [5] (we have chosen this method because it has been published recently, hence it is expectable that it is competitive with respect to other ones). The method in [5] allows one to solve the original optimization problem (5) in the unconstrained case, i.e., $n_{G}=0$.

In [5], the positive definiteness of the trigonometric matrix polynomial $F(z)$ is ensured by looking for a scalar $\mu>0$ and a Gram matrix $Z=Z^{H}$ satisfying the LMI

$$
\left\{\begin{array}{c}
\left(\begin{array}{cc}
\Re(Z) & \Im(Z) \\
\star & \Re(Z)
\end{array}\right)-\mu I \geq 0 \\
F(z)=(I \otimes h(z))^{H} Z(I \otimes h(z))
\end{array}\right.
$$

where $h(z)$ is a vector containing all monomials in $z$ of degree not greater than $d_{h v}$, where the integer $d_{h v}$ satisfies $d_{h v} \geq \operatorname{deg}(F)$. Let us observe that this method does not require to substitute the complex variable $z$ with its parametrization (16) obtained through the real variables $x$ and $y$.

For the proposed approach, the LMI certifying the SOS condition (27) is built as explained in Section 2.2. The vector of monomials $b(v)$ used to define the Gram matrix of $A(v)$ contains all monomials in $v$ of degree not greater than $d$ that satisfy (31), where the integer $d$ satisfies $d \geq\lceil\operatorname{deg}(F) / 2\rceil$. The multipliers $Q_{l}(v)$ have degree $2(d-1)$ and satisfy $(26)$.

Theorem 3 Let us consider the case of unconstrained trigonometric polynomials (i.e., $m=1$ and $n_{G}=0$ ). For the method in [5], the size of the 
LMI and the number of independent real LMI variables in (38) are $\zeta_{h v} \times \zeta_{h v}$ and $\eta_{h v}$, where

$$
\zeta_{h v}=2 \frac{\left(n+d_{h v}\right) !}{n ! d_{h v} !}
$$

and

$$
\eta_{h v}=\frac{1}{4} \zeta_{h v}^{2}-\phi_{1}+1
$$

where $\phi_{1}$ is the number of vectors $k \in \mathbb{Z}^{n}$ that satisfy

$$
\left\{\begin{array}{l}
k=k^{+}-k^{-} \\
k^{+}, k^{-} \in \mathbb{N}^{n} \\
k_{1}^{+}+\ldots+k_{n}^{+} \leq d_{h v} \\
k_{1}^{-}+\ldots+k_{n}^{-} \leq d_{h v} .
\end{array}\right.
$$

For the proposed approach in Section 3.1, the size of the LMI and the number of independent real LMI variables are $\zeta \times \zeta$ and $\eta$, where

$$
\zeta=\sum_{i=0}^{\min \{n, d\}} \frac{(n+d-i) !}{n !(d-i) !} \frac{n !}{(n-i) ! i !}
$$

and

$$
\eta=\frac{1}{2} \zeta(\zeta+1)-\phi_{2}(n, d)+n \phi_{2}(n-1, d-1)+1
$$

where $\phi_{2}(n, d)$ is the number of vectors $k \in \mathbb{N}^{2 n}$ that satisfy

$$
\left\{\begin{array}{l}
k_{\sigma} \leq 2 \quad \forall \sigma=n+1, \ldots, 2 n \\
k_{1}+\ldots+k_{2 n} \leq 2 d
\end{array}\right.
$$

Proof. First, (39) holds because $\zeta_{h v}$ is the number of monomials in $n$ variables of degree not greater than $d_{h v}$, which has to be doubled since the real LMI has double size than the complex Gram matrix $Z$ from (38). Then, (40) holds because the number of independent real LMI variables is the number of independent entries of $Z$, i.e. $\zeta_{h v}^{2} / 4$, minus the number of monomials (in the extended variable $(z, \overline{(} z))$ ) that can be generated by the product $h(z)^{H} Z h(z)$ for some $Z$, i.e. $\phi_{1}$, plus one for $\mu$.

Next, (42) holds because $\zeta$ is the number of monomials in $2 n$ variables of degree not greater than $d$ where $n$ variables have powers not greater than 1 (in particular, $\frac{(n+d-i) !}{n !(d-i) !}$ is the number of monomials in $n$ variables of degree not greater than $d-i$, and $\frac{n !}{(n-i) ! i !}$ is the number of monomials in $n$ variables of degree $i$ with powers not greater than 1). Then, (43) holds because 
the number of independent real LMI variables is the number of independent entries of the Gram matrix of $A(v)$, i.e. $\zeta(\zeta+1) / 2$, minus the number of monomials that can be generated by the product $b(v)^{T} C b(v)$ for some $C$, i.e. $\phi_{2}(n, d)$, plus the independent coefficients of the matrix polynomials $Q_{l}(v)$, i.e. $n \phi_{2}(n-1, d-1)$, plus one for $\mu$.

Tables 1-2 show the quantities $\zeta_{h v}, \eta_{h v}, \zeta$ and $\eta$ found in Theorem 3. The degrees $d_{h v}$ of the method in [5] and $d$ of the proposed approach are set in order to generate trigonometric polynomials of the same degree $\operatorname{deg}(F)$, i.e.

\begin{tabular}{|c|c|c|c|c|c|c|}
\hline$\left\{d_{h}\right.$ & $\begin{array}{c}h v \\
d\end{array}$ & $\begin{array}{l}=1 \\
=1\end{array}$ & $\begin{array}{l}\operatorname{leg}(F \\
\operatorname{deg}(\end{array}$ & $\begin{array}{l}\text { ) } \\
F) / 2\rceil\end{array}$ & & \\
\hline$n \backslash \operatorname{deg}(F)$ & 1 & 2 & 3 & 4 & 5 & 6 \\
\hline 1 & 4 & 6 & 8 & 10 & 12 & 14 \\
\hline 2 & 6 & 12 & 20 & 30 & 42 & 56 \\
\hline 3 & 8 & 20 & 40 & 70 & 112 & 168 \\
\hline 4 & 10 & 30 & 70 & 140 & 252 & 420 \\
\hline & & $\left(\zeta_{h}\right.$ & & & & \\
\hline$n \backslash \operatorname{deg}(F)$ & 1 & 2 & 3 & 4 & 5 & 6 \\
\hline 1 & 3 & 3 & 5 & 5 & 7 & 7 \\
\hline 2 & 5 & 5 & 13 & 13 & 25 & 25 \\
\hline 3 & 7 & 7 & 25 & 25 & 63 & 63 \\
\hline 4 & 9 & 9 & 41 & 41 & 129 & 129 \\
\hline
\end{tabular}

$(\zeta)$

Table 1: Size of the LMI for the method in [5] $\left(\zeta_{h v}\right)$ and for the proposed approach $(\zeta)$.

\section{Examples}

This section provides some illustrative examples of the proposed conditions. The SDPs are solved with the toolbox SeDuMi for Matlab [19] on a standard PC (Windows 7, Intel Core 2, $3 \mathrm{GHz}, 4 \mathrm{~GB}$ RAM). 


\begin{tabular}{c|cccccc}
$n \backslash \operatorname{deg}(F)$ & 1 & 2 & 3 & 4 & 5 & 6 \\
\hline 1 & 2 & 5 & 10 & 17 & 26 & 37 \\
2 & 3 & 18 & 64 & 165 & 351 & 658 \\
3 & 4 & 46 & 254 & 917 & 2576 & 6134 \\
4 & 5 & 95 & 755 & 3650 & 13126 & 38780 \\
\multicolumn{7}{c}{$\left(\eta_{h v}\right)$} \\
$n \backslash \operatorname{deg}(F)$ & 1 & 2 & 3 & 4 & 5 & 6 \\
\hline 1 & 2 & 2 & 7 & 7 & 16 & 16 \\
2 & 3 & 3 & 52 & 52 & 247 & 247 \\
3 & 4 & 4 & 200 & 200 & 1684 & 1684 \\
4 & 5 & 5 & 547 & 547 & 7261 & 7261 \\
& & & $(\eta)$ & & &
\end{tabular}

Table 2: Number of independent real LMI variables for the method in [5] $\left(\eta_{h v}\right)$ and for the proposed approach $(\eta)$.

\subsection{Example 1}

Let us start by considering (5) with $n=4, n_{G}=0$, and

$$
\left\{\begin{array}{l}
F(z)=E(z)+E(z)^{H} \\
E(z)=j z_{1} z_{2} z_{3} z_{4}+\sum_{i=1}^{4}\left(i z_{i}+z_{i}^{4}\right) .
\end{array}\right.
$$

First, we compute the lower bound $\hat{\mu}$ in (32). The degree of the multipliers $Q_{l}(v)$ and the vector of monomials used to define the Gram matrix of $A(v)$ are chosen as explained in Section 3.2 with the smallest admissible degree $d$ (i.e., $d=2$ ). We find

$$
\hat{\mu}=-24.966 .
$$

The size of the LMI is $41 \times 41$, the number of independent real LMI variables is 547 , and the computational time is 1.1 seconds.

Second, let us establish if the found lower bound is tight. We find that (36) holds with

$$
\begin{aligned}
v= & (-0.734,-0.760,-0.784,-0.812, \\
& -0.680,-0.650,-0.621,0.584)^{T} .
\end{aligned}
$$

Hence, from Theorem 2 we conclude that $\hat{\mu}$ is tight, and $\xi(v)$ is a minimizer of the original optimization problem (5). 
For comparison we test the method in [5] solving (38) with the smallest admissible degree $d_{h v}$ (i.e., $d_{h v}=4$ ), however our Matlab runs out of memory (the number of independent real LMI variables is 3650 as shown by Table $2)$.

\subsection{Example 2}

Here we consider an application in the estimation of reduced order models. Specifically, the problem consists of determining a polynomial $\hat{p}(z)$ in a chosen class $\mathcal{A}$ that approximates a given polynomial $p(z)$ on a region $\mathcal{G}$ of $\mathcal{T}^{n}$ to the best achievable accuracy, i.e.,

$$
\begin{aligned}
& \gamma^{*}= \inf _{\gamma, \hat{p}(z)} \gamma \\
& \text { s.t. }\left\{\begin{array}{l}
|p(z)-\hat{p}(z)|<\gamma \quad \forall z \in \mathcal{G} \\
\hat{p}(z) \in \mathcal{A} .
\end{array}\right.
\end{aligned}
$$

We choose

$$
\left\{\begin{aligned}
p(z)= & 0.4+j 0.1 z_{2}+0.3 z_{1}^{2}-0.4 z_{1} z_{2}+0.2 z_{1}^{4}+j 0.3 z_{2}^{4} \\
\mathcal{G}= & \left\{z \in \mathcal{T}^{2}: \Re\left(z_{1}\right) \geq 0.5\right\} \\
\mathcal{A}: & \text { set of polynomials in } z \text { of degree } 1 \\
& \text { with real coefficients. }
\end{aligned}\right.
$$

Let us observe that the condition $|p(z)-\hat{p}(z)|<\gamma$ for all $z \in \mathcal{G}$ can be equivalently rewritten as

$$
F(z)>0 \quad \forall z \in \mathcal{G}
$$

by defining

$$
F(z)=\left(\begin{array}{cc}
\gamma & p(z)-\hat{p}(z) \\
\star & \gamma
\end{array}\right) .
$$

Moreover, the region $\mathcal{G}$ can be expressed as in (6) by choosing $n_{G}=1$ and

$$
g_{1}(z)=\left(z_{1}+z_{1}^{-1}-1\right)\left(3-z_{1}-z_{1}^{-1}\right)
$$

(observe that this choice of $g_{1}(z)$ ensures that Assumption 1 holds).

First, we solve the LMI feasibility test (26)-(31) letting $\gamma$ and the coefficients of $\hat{p}(z)$ be variables. The degree of the multipliers $P_{i}(v)$ and $Q_{l}(v)$ are chosen as the largest ones for which $A(v)$ has its minimum degree. We find the upper bound of $\gamma^{*}$ given by

$$
\hat{\gamma}=0.913
$$


which is achieved by

$$
\hat{p}(z)=0.193+0.263 z_{1}-0.253 z_{2} .
$$

The sizes of the LMIs are $52 \times 52$ and $20 \times 20$, the number of independent real LMI variables is 620 , and the computational time is 1.5 seconds.

Second, by following a strategy similar to the one given in Theorem 2, we find that this upper bound is tight. Indeed,

$$
z=\left(\begin{array}{c}
0.989-j 0.149 \\
-0.450-j 0.893
\end{array}\right) \Rightarrow|p(z)-\hat{p}(z)|=\hat{\gamma}
$$

It is interesting to observe that, removing the constraint $\Re\left(z_{1}\right) \geq 0.5$ (i.e., considering $\mathcal{G}=\mathcal{T}^{2}$ ), the solution would change. In particular, one would obtain $\hat{\gamma}=1.171$.

\section{Conclusions}

The contribution of this paper is to show that, by exploiting Putinar's Positivstellensatz and introducing suitable transformations, it is possible to derive a nonconservative approach based on SDP whose computational burden can be significantly smaller than that of an existing method recently published. Other advantages of the proposed approach include the possibility of taking into account the presence of constraints in the form of semi-algebraic sets and establishing tightness of a found lower bound.

\section{Acknowledgements}

The author would like to thank the Associate Editor and the Reviewers for their useful comments. This work is supported in part by the Research Grants Council of Hong Kong under Grant HKU711213E.

\section{References}

[1] K. S. Narendra and J. H. Taylor. Frequency domain criteria for absolute stability. Academic Press Inc., New York, 1973.

[2] L. Qiu and K. Zhou. An Introduction to Feedback Control. Prentice Hall, 2010. 
[3] S. Boyd, L. El Ghaoui, E. Feron, and V. Balakrishnan. Linear Matrix Inequalities in System and Control Theory. SIAM, 1994.

[4] T. Mora. Solving Polynomial Equation Systems I. Cambridge University Press, 2003.

[5] D. Henrion and T. Vyhlidal. Positive trigonometric polynomials for strong stability of difference equations. Automatica, 48(9):2207-2212, 2012 .

[6] T. Roh, B. Dumitrescu, and L. Vanderberghe. Multidimensional FIR filter design via trigonometric sum-of-squares optimization. IEEE Journal on Selected Topics in Signal Processing, 1(4):641-650, 2007.

[7] G. Chesi, A. Tesi, A. Vicino, and R. Genesio. On convexification of some minimum distance problems. In European Control Conference, Karlsruhe, Germany, 1999.

[8] P. A. Parrilo. Structured semidefinite programs and semialgebraic geometry methods in robustness and optimization. $\mathrm{PhD}$ thesis, California Institute of Technology, 2000.

[9] J.-B. Lasserre. Global optimization with polynomials and the problem of moments. SIAM Journal of Optimization, 11(3):796-817, 2001.

[10] M. Kojima. Sums of squares relaxations of polynomial semidefinite programs. Technical report, Tokyo Institute of Technology, 2003.

[11] S. Prajna, A. Papachristodoulou, and F. Wu. Nonlinear control synthesis by sum of squares optimization: a Lyapunov-based approach. In Asian Control Conference, 2004.

[12] C. W. J. Hol and C. W. Scherer. Computing optimal fixed order $H_{\infty}$ synthesis values by matrix sum of squares relaxations. In IEEE Conference on Decision and Control, pages 3147-3153, Paradise Island, Bahamas, 2004.

[13] C. W. Scherer and C. W. J. Hol. Matrix sum-of-squares relaxations for robust semi-definite programs. Mathematical Programming Series B, 107(1-2):189-211, 2006.

[14] G. Chesi. LMI techniques for optimization over polynomials in control: a survey. IEEE Transactions on Automatic Control, 55(11):2500-2510, 2010 . 
[15] M. Putinar. Positive polynomials on compact semi-algebraic sets. Ind. Univ. Math., 42:969-984, 1993.

[16] D. Henrion and J.-B. Lasserre. Detecting global optimality and extracting solutions in GloptiPoly. In D. Henrion and A. Garulli, editors, Positive Polynomials in Control. Springer, 2005.

[17] G. Chesi. Domain of Attraction: Analysis and Control via SOS Programming. Springer, 2011.

[18] J. Nie. Certifying convergence of lasserre's hierarchy via flat truncation. Mathematical Programming Ser. A, 142(1-2):485-510, 2013.

[19] J. F. Sturm. Using SeDuMi 1.02, a MATLAB toolbox for optimization over symmetric cones. Optimization Methods and Software, 11-12:625653, 1999. 